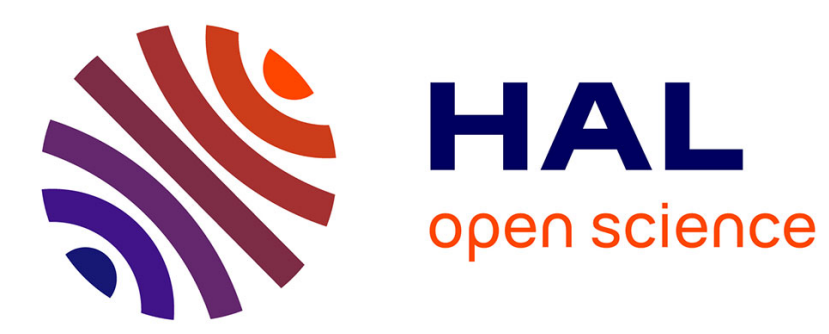

\title{
Adaptive Parameterized Consistency
}

Amine Balafrej, Christian Bessiere, Remi Coletta, El Houssine Bouyakhf

\section{To cite this version:}

Amine Balafrej, Christian Bessiere, Remi Coletta, El Houssine Bouyakhf. Adaptive Parameterized Consistency. CP: Principles and Practice of Constraint Programming, Sep 2013, Uppsala, Sweden. pp.143-158, 10.1007/978-3-642-40627-0_14 . lirmm-00839025

\section{HAL Id: lirmm-00839025 https://hal-lirmm.ccsd.cnrs.fr/lirmm-00839025}

Submitted on 23 Jan 2018

HAL is a multi-disciplinary open access archive for the deposit and dissemination of scientific research documents, whether they are published or not. The documents may come from teaching and research institutions in France or abroad, or from public or private research centers.
L'archive ouverte pluridisciplinaire HAL, est destinée au dépôt et à la diffusion de documents scientifiques de niveau recherche, publiés ou non, émanant des établissements d'enseignement et de recherche français ou étrangers, des laboratoires publics ou privés. 


\title{
Adaptive Parameterized Consistency ${ }^{\star}$
}

\author{
Amine Balafrej ${ }^{1,2}$, Christian Bessiere ${ }^{1}$, Remi $_{\text {Coletta }}{ }^{1}$, El Houssine Bouyakhf ${ }^{2}$ \\ ${ }^{1}$ CNRS, University of Montpellier, France \\ ${ }^{2}$ LIMIARF/FSR, University Mohammed V Agdal, Rabat, Morocco \\ $\{$ balafrej, bessiere, coletta\}@lirmm.fr, bouyakhf@fsr.ac.ma
}

\begin{abstract}
State-of-the-art constraint solvers uniformly maintain the same level of local consistency (usually arc consistency) on all the instances. We propose parameterized local consistency, an original approach to adjust the level of consistency depending on the instance and on which part of the instance we propagate. We do not use as parameter one of the features of the instance, as done for instance in portfolios of solvers. We use as parameter the stability of values, which is a feature based on the state of the arc consistency algorithm during its execution. Parameterized local consistencies choose to enforce arc consistency or a higher level of local consistency on a value depending on whether the stability of the value is above or below a given threshold. We also propose a way to dynamically adapt the parameter, and thus the level of local consistency, during search. This approach allows us to get a good trade-off between the number of values pruned and the computational cost. We validate our approach on various problems from the CSP competition.
\end{abstract}

\section{Introduction}

Enforcing constraint propagation by applying local consistency during search is one of the strengths of constraint programming $(\mathrm{CP})$. It allows the constraint solver to remove locally inconsistent values. This leads to a reduction of the search space. Arc consistency is the oldest and most well-known way of propagating constraints [2]. It has the nice feature that it does not modify the structure of the constraint network. It just prunes infeasible values. Arc consistency is the standard level of consistency maintained in constraint solvers. Several other local consistencies pruning only values and stronger than arc consistency have been proposed, such as max restricted path consistency or singleton arc consistency [7]. These local consistencies are seldom used in practice because of the high computational cost of maintaining them during search. However, on some problems, maintaining arc consistency is not a good choice because of the high number of ineffective revisions of constraints that penalize the CPU time. For instance, Stergiou observed that when solving the scen11 radio link frequency assignment problem (RLFAP) with an algorithm maintaining arc consistency, only 27 out of the 4103 constraints of the problem were identified as causing a domain wipe out and 1921 constraints did not prune any value [10].

\footnotetext{
* This work has been funded by the EU project ICON (FP7-284715).
} 
Choosing the right level of local consistency for solving a problem requires finding the good trade-off between the ability of this local consistency to remove inconsistent values, and the cost of the algorithm that enforces it. Stergiou suggests to take advantage of the power of strong consistencies to reduce the search space while avoiding the high cost of maintaining them in the whole network. His method results in a heuristic approach based on the monitoring of propagation events to dynamically adapt the level of local consistency (arc consistency or max restricted path consistency) to individual constraints. This prunes more values than arc consistency and less than max restricted path consistency. The level of propagation obtained is not characterized by a local consistency property. Depending on the order of propagation we can converge on different closures. When dealing with global constraints, some work propose to weaken arc consistency instead of strengthening it. In [8], Katriel et al. proposed a randomized filtering scheme for AllDifferent and Global Cardinality Constraint. In [9], Sellmann introduced the concept of approximated consistency for optimization constraints and provided filtering algorithms for Knapsack Constraints based on bounds with guaranteed accuracy.

In this paper we define the notion of stability of values. This is an original notion not based on characteristics of the instance to solve but based on the state of the arc consistency algorithm during its propagation. Based on this notion, we propose parameterized consistencies, an original approach to adjust the level of consistency inside a given instance. The intuition is that if a value is hard to prove arc consistent (i.e., the value is not stable for arc consistency), this value will perhaps be pruned by a stronger local consistency. The parameter $p$ specifies the threshold of stability of a value $v$ below which we will enforce a higher consistency to $v$. A parameterized consistency $p$-LC is thus an intermediate level of consistency between arc consistency and another consistency LC, stronger than arc consistency. The strength of $p$-LC depends on the parameter $p$. This approach allows us to find a trade-off between the pruning power of the local consistency and the computational cost of the algorithm that achieves it. We apply $p$-LC to the case where LC is max restricted path consistency. We describe the algorithm $p$-maxRPC3 (based on maxRPC3 [1]) that achieves $p$-max restricted path consistency. Then, we propose $a p$ - $\mathrm{LC}$, an adaptive variant of $p$-LC which adapts dynamically and locally the level of local consistency during search. Finally, we experimentally assess the practical relevance of parameterized local consistency. We show that by making good choices for the parameter $p$ we take advantage of both arc consistency light computational cost and LC effectiveness of pruning. In the best cases, a solver using $p$-LC explores the same number of nodes as LC with a number of constraint checks lower than LC, resulting in a CPU-time lower than both arc consistency-based or LC-based solvers.

\section{Background}

A constraint network is defined as a set of $n$ variables $X=\left\{x_{1}, \ldots, x_{n}\right\}$, a set of ordered domains $D=\left\{D\left(x_{1}\right), \ldots, D\left(x_{n}\right)\right\}$, and a set of $e$ constraints 
$C=\left\{c_{1}, \ldots, c_{e}\right\}$. Each constraint $c_{k}$ is defined by a pair $\left(\operatorname{var}\left(c_{k}\right), \operatorname{sol}\left(c_{k}\right)\right)$, where $\operatorname{var}\left(c_{k}\right)$ is an ordered subset of $X$, and $\operatorname{sol}\left(c_{k}\right)$ is a set of combinations of values (tuples) satisfying $c_{k}$. In the following, we restrict ourselves to binary constraints because the local consistency (maxRPC) we use here to instantiate our approach is defined on the binary case only. However, the notions we introduce can be extended to non-binary constraints, by using maxRPWC for instance [4]. A binary constraint $c$ between $x_{i}$ and $x_{j}$ will be denoted by $c_{i j}$, and $\Gamma\left(x_{i}\right)$ will denote the set of variables $x_{j}$ involved in a constraint with $x_{i}$.

A value $v_{j} \in D\left(x_{j}\right)$ is called an arc consistent support (AC support) for $v_{i} \in D\left(x_{i}\right)$ on $c_{i j}$ if $\left(v_{i}, v_{j}\right) \in \operatorname{sol}\left(c_{i j}\right)$. A value $v_{i} \in D\left(x_{i}\right)$ is arc consistent (AC) if and only if for all $x_{j} \in \Gamma\left(x_{i}\right) v_{i}$ has an AC support $v_{j} \in D\left(x_{j}\right)$ on $c_{i j}$. A domain $D\left(x_{i}\right)$ is arc consistent if it is non empty and all values in $D\left(x_{i}\right)$ are arc consistent. A network is arc consistent if all domains in $D$ are arc consistent. If enforcing arc consistency on a network $N$ leads to a domain wipe out, we say that $N$ is arc inconsistent.

A tuple $\left(v_{i}, v_{j}\right) \in D\left(x_{i}\right) \times D\left(x_{j}\right)$ is path consistent $(P C)$ if and only if for any third variable $x_{k}$ there exists a value $v_{k} \in D\left(x_{k}\right)$ such that $v_{k}$ is an AC support for both $v_{i}$ and $v_{j}$. In such a case, $v_{k}$ is called witness for the path consistency of $\left(v_{i}, v_{j}\right)$.

A value $v_{j} \in D\left(x_{j}\right)$ is a max restricted path consistent (maxRPC) support for $v_{i} \in D\left(x_{i}\right)$ on $c_{i j}$ if and only if it is an AC support and the tuple $\left(v_{i}, v_{j}\right)$ is path consistent. A value $v_{i} \in D\left(x_{i}\right)$ is max restricted path consistent on a constraint $c_{i j}$ if and only if $\exists v_{j} \in D\left(x_{j}\right)$ maxRPC support for $v_{i}$ on $c_{i j}$. A value $v_{i} \in D\left(x_{i}\right)$ is max restricted path consistent iff for all $x_{j} \in \Gamma\left(x_{i}\right) v_{i}$ has a maxRPC support $v_{j} \in D\left(x_{j}\right)$ on $c_{i j}$. A domain $D\left(x_{i}\right)$ is maxRPC if it is non empty and all values in $D\left(x_{i}\right)$ are maxRPC. A network is maxRPC if all domains in $D$ are maxRPC.

We say that a local consistency $L C_{1}$ is stronger than a local consistency $L C_{2}$ ( $L C_{2} \preceq L C_{1}$ ) if $L C_{2}$ holds on any constraint network on which $L C_{1}$ holds.

The problem of deciding whether a constraint network has solutions is called the constraint satisfaction problem (CSP), and it is NP-complete. Solving a CSP is done by backtrack search that maintains some level of consistency between each branching step.

\section{Parameterized Consistency}

In this section we present an original approach to parameterize a level of consistency LC stronger than arc consistency so that it degenerates to arc consistency when the parameter equals 0 , to LC when the parameters equals 1 , and to levels in between when the parameter is between 0 and 1 . The idea behind this is to be able to adjust the level of consistency to the instance to be solved, hoping that such an adapted level of consistency will prune significantly more values than arc consistency while being less time consuming than LC.

Parameterized consistency is based on the concept of stability of values. We first need to define the 'distance to end' of a value in a domain. This captures 


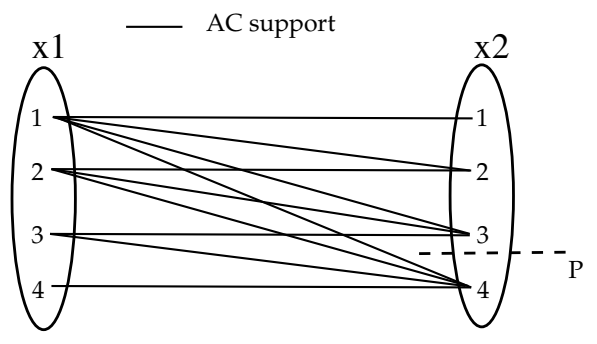

Fig. 1. Stability of supports on the example of the constraint $x_{1} \leq x_{2}$ with the domains $D\left(x_{1}\right)=D\left(x_{2}\right)=\{1,2,3,4\} .\left(x_{1}, 4\right)$ is not p-stable for AC.

how far a value is from the last in its domain. In the following, $\operatorname{rank}(v, S)$ is the position of value $v$ in the ordered set of values $S$.

Definition 1 (Distance to end of a value). The distance to end of a value $v_{i} \in D\left(x_{i}\right)$ is the ratio

$$
\Delta\left(x_{i}, v_{i}\right)=\left(\left|D_{o}\left(x_{i}\right)\right|-\operatorname{rank}\left(v_{i}, D_{o}\left(x_{i}\right)\right)\right) /\left|D_{o}\left(x_{i}\right)\right|,
$$

where $D_{o}\left(x_{i}\right)$ is the initial domain of $x_{i}$.

We see that the first value in $D_{o}\left(x_{i}\right)$ has distance $\left(\left|D_{o}\left(x_{i}\right)\right|-1\right) /\left|D_{o}\left(x_{i}\right)\right|$ and the last one has distance 0 . Thus, $\forall v_{i} \in D\left(x_{i}\right), 0 \leq \Delta\left(x_{i}, v_{i}\right)<1$.

We can now give the definition of what we call the parameterized stability of a value for arc consistency. The idea is to define stability for values based on the distance to the end of their AC supports. For instance, consider the constraint $x_{1} \leq x_{2}$ with the domains $D\left(x_{1}\right)=D\left(x_{2}\right)=\{1,2,3,4\}$ (see Figure 1). $\Delta\left(x_{2}, 1\right)=(4-1) / 4=0.75, \Delta\left(x_{2}, 2\right)=0.5, \Delta\left(x_{2}, 3\right)=0.25$ and $\Delta\left(x_{2}, 4\right)=0$. If $p=0.2$, the value $\left(x_{1}, 4\right)$ is not $p$-stable for $\mathrm{AC}$, because the first and only $\mathrm{AC}$ support of $\left(x_{1}, 4\right)$ in the ordering used to look for supports, that is $\left(x_{2}, 4\right)$, has a distance to end smaller than the threshold $p$. Proving that the pair $(4,4)$ is inconsistent (by a stronger consistency) could lead to the pruning of $\left(x_{1}, 4\right)$. In other words, applying a stronger consistency on $\left(x_{1}, 4\right)$ has more chances to lead to its removal than applying it on for instance $\left(x_{1}, 1\right)$, which had no difficulty to find its first AC support (distance to en of $\left(x_{2}, 1\right)$ is 0.75 ).

Definition 2 ( $p$-stability for AC). A value $v_{i} \in D\left(x_{i}\right)$ is p-stable for $A C$ on $c_{i j}$ iff $v_{i}$ has an $A C$ support $v_{j} \in D\left(x_{j}\right)$ on $c_{i j}$ such that $\Delta\left(x_{j}, v_{j}\right) \geq p$. A value $v_{i} \in D\left(x_{i}\right)$ is p-stable for $A C$ iff $\forall x_{j} \in \Gamma\left(x_{i}\right), v_{i}$ is p-stable for $A C$ on $c_{i j}$.

We are now ready to give the first definition of parameterized local consistency. This first definition can be applied to any local consistency LC for which the consistency of a value on a constraint is well defined. This is the case for instance for all triangle-based consistencies [6,2]. 
Definition 3 (Constraint-based $p$-LC). Let $L C$ be a local consistency stronger than $A C$ for which the $L C$ consistency of a value on a constraint is defined. A value $v_{i} \in D\left(x_{i}\right)$ is constraint-based $p$-LC on $c_{i j}$ iff it is p-stable for $A C$ on $c_{i j}$, or it is $L C$ on $c_{i j}$. A value $v_{i} \in D\left(x_{i}\right)$ is constraint-based $p$-LC iff $\forall c_{i j}, v_{i}$ is constraint-based $p-L C$ on $c_{i j}$. A constraint network is constraint-based $p$-LC iff all values in all domains in $D$ are constraint-based $p-L C$.

Theorem 1. Let $L C$ be a local consistency stronger than $A C$ for which the $L C$ consistency of a value on a constraint is defined. Let $p_{1}$ and $p_{2}$ be two parameters in [0..1]. If $p_{1}<p_{2}$ then $A C \preceq$ constraint-based $p_{1}-L C \preceq$ constraint-based $p_{2}$ $L C \preceq L C$.

Proof. Suppose that there exist two parameters $p_{1}, p_{2}$ such that $0 \leq p_{1}<p_{2} \leq$ 1 , and suppose that there exists a $p_{2}$-LC constraint network $N$ that contains a $p_{2}$-LC value $\left(x_{i}, v_{i}\right)$ that is $p_{1}$-LC inconsistent. Let $c_{i j}$ be the constraint on which $\left(x_{i}, v_{i}\right)$ is $p_{1}$-LC inconsistent. Then, $\nexists v_{j} \in D\left(x_{j}\right)$ that is an AC support for $\left(x_{i}, v_{i}\right)$ on $c_{i j}$ such that $\Delta\left(x_{j}, v_{j}\right) \geq p_{1}$. Thus, $v_{i}$ is not $p_{2}$-stable for $\mathrm{AC}$ on $c_{i j}$. In addition, $v_{i}$ is not $\mathrm{LC}$ on $c_{i j}$. Therefore, $v_{i}$ is not $p_{2}-\mathrm{LC}$, and $N$ is not $p_{2}$-LC.

Definition 3 can be modified to a more coarse-grained version that is not dependent on the consistency of values on a constraint. It will have the advantage to apply to any type of strong local consistency, even those, like singleton arc consistency, for which the consistency of a value on a constraint is not defined.

Definition 4 (Value-based $p$-LC). Let LC be a local consistency stronger than $A C$. A value $v_{i} \in D\left(x_{i}\right)$ is value-based $p$ - $L C$ if and only if it is p-stable for $A C$ or it is LC. A constraint network is value-based $p-L C$ if and only if all values in all domains in $D$ are value-based $p-L C$.

Theorem 2. Let LC be a local consistency stronger than AC. Let $p_{1}$ and $p_{2}$ be two parameters in [0..1]. If $p_{1}<p_{2}$ then $A C \preceq$ value-based $p_{1}-L C \preceq$ value-based $p_{2}-L C \preceq L C$.

Proof. Suppose that there exist two parameters $p_{1}, p_{2}$ such that $0 \leq p_{1}<p_{2} \leq$ 1 , and suppose that there exists a $p_{2}$-LC constraint network $N$ that contains a $p_{2}$-LC value $\left(x_{i}, v_{i}\right)$ that is $p_{1}$-LC-inconsistent. $v_{i}$ is $p_{1}$-LC-inconsistent means that:

1. $v_{i}$ is not $p_{1}$-stable for AC: $\exists c_{i j}$ on which $v_{i}$ is not $p_{1}$-stable for AC. Then $\nexists v_{j} \in D\left(x_{j}\right)$ that is an AC support for $\left(x_{i}, v_{i}\right)$ on $c_{i j}$ such that $\Delta\left(x_{j}, v_{j}\right) \geq p_{1}$. Therefore, $v_{i}$ is not $p_{2}$-stable for $\mathrm{AC}$ on $c_{i j}$, then $v_{i}$ is not $p_{2}$-stable for AC. 2. $v_{i}$ is LC inconsistent

(1) and (2) imply that $v_{i}$ is not $p_{2}-\mathrm{LC}$, and $N$ is not $p_{2}-\mathrm{LC}$.

For both types of definitions of $p$-LC, we have the following property on the extreme cases $(p=0, p=1)$.

Corollary 1. Let $L C_{1}$ and $L C_{2}$ be two local consistencies stronger than $A C$. We have: value-based $0-L C_{2}=A C$ and value-based $1-L C_{2}=L C$. If the $L C_{1}$ consistency of a value on a constraint is defined, we also have: constraint-based $0-L C_{1}=A C$ and constraint-based 1-LC $=L C$. 


\section{Parameterized maxRPC: $p$-maxRPC}

To illustrate the benefit of our approach, we apply parameterized consistency to maxRPC to obtain the $p$-maxRPC level of consistency that achieves a consistency level between $\mathrm{AC}$ and maxRPC.

Definition 5 ( $p$-maxRPC). A value, a network, are $p$-maxRPC if and only if they are constraint-based $p$-maxRPC.

From Theorem 1 and Corollary 1 we derive the following corollary.

Corollary 2. For any two parameters $p_{1}, p_{2}, 0 \leq p_{1}<p_{2} \leq 1, A C \preceq p_{1}$ $\max R P C \preceq p_{2}-\max R P C \preceq \max R P C .0-\max R P C=A C$ and $1-\max R P C=$ $\max R P C$.

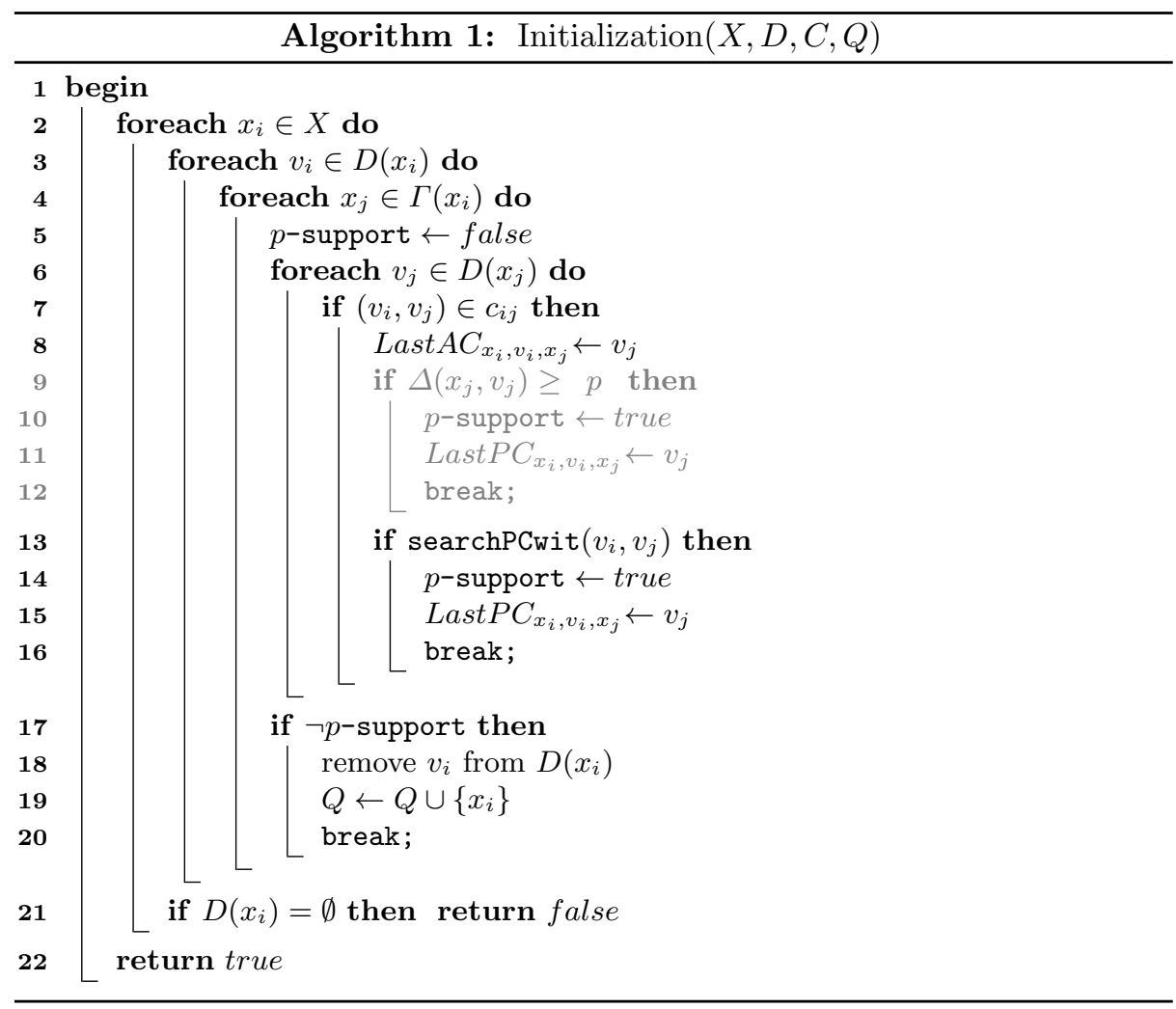

We propose an algorithm for $p$-maxRPC, based on maxRPC3, the best existing maxRPC algoritm. We do not describe maxRPC3 in full detail as it can be found in [1]. We only describe procedures where changes to maxRPC3 are 

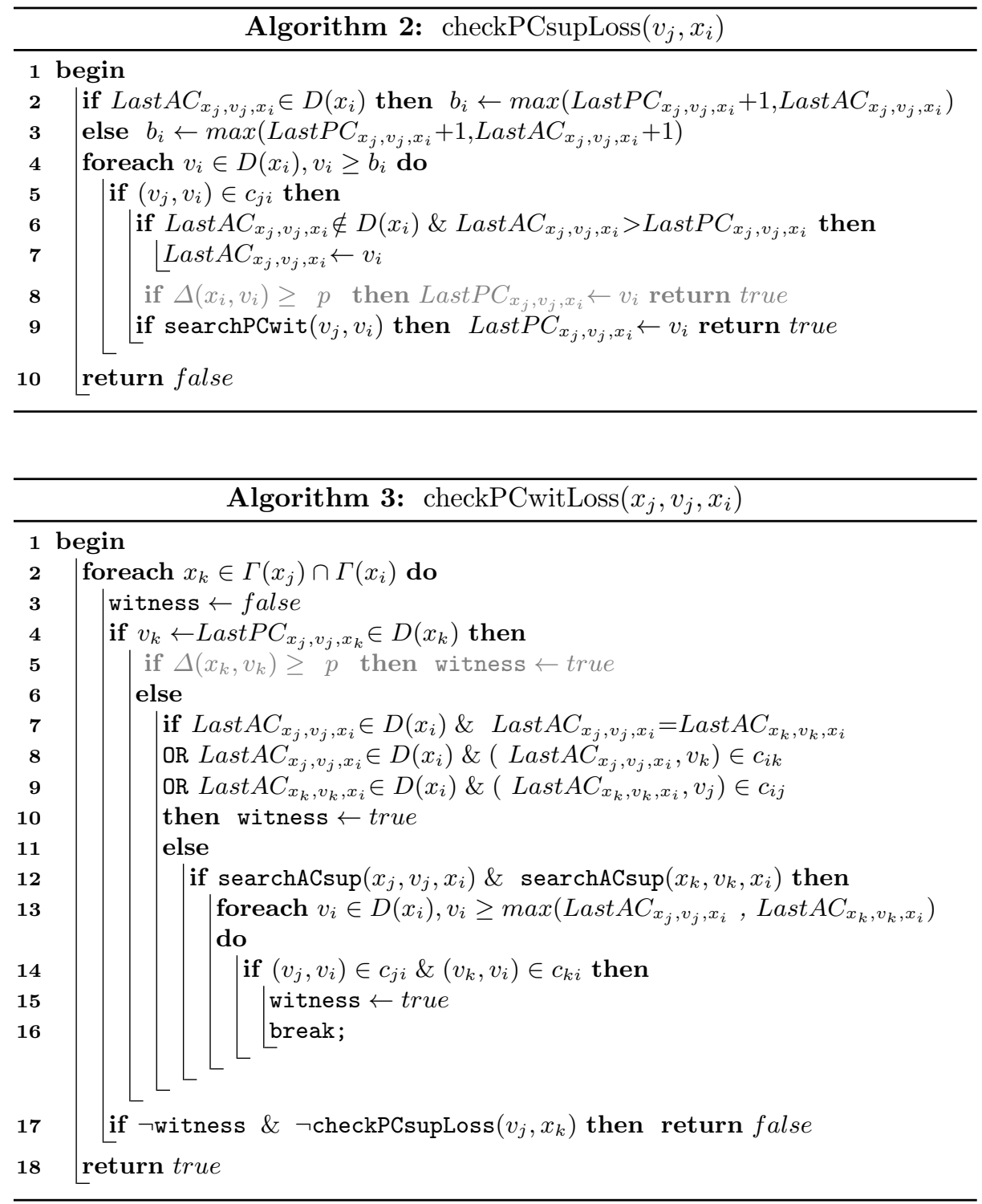

necessary to design $p$-maxRPC3, a coarse grained algorithm that performs $p$ maxRPC. We use light grey to emphasize the modified parts of the original maxRPC3 algorithm.

maxRPC3 uses a propagation list $Q$ where it inserts the variables whose domains have changed. It also uses two other data structures: LastAC and LastPC. For each value $\left(x_{i}, v_{i}\right)$ Last $\mathrm{AC}_{x_{i}, v_{i}, x_{j}}$ stores the smallest AC support for $\left(x_{i}, v_{i}\right)$ on $c_{i j}$ and $\mathrm{LastPC}_{x_{i}, v_{i}, x_{j}}$ stores the smallest PC support for $\left(x_{i}, v_{i}\right)$ on $c_{i j}$ (i.e., 
the smallest AC support $\left(x_{j}, v_{j}\right)$ for $\left(x_{i}, v_{i}\right)$ on $c_{i j}$ such that $\left(v_{i}, v_{j}\right)$ is $\left.\mathrm{PC}\right)$. This algorithm consists in two phases: initialization and propagation.

In the initialization phase (algorithm 1) maxRPC3 checks if each value $\left(x_{i}, v_{i}\right)$ has a maxRPC-support $\left(x_{j}, v_{j}\right)$ on each constraint $c_{i j}$. If not, it removes $v_{i}$ from $D\left(x_{i}\right)$ and inserts $x_{i}$ in $Q$. To check if a value $\left(x_{i}, v_{i}\right)$ has a maxRPC-support on a constraint $c_{i j}$, maxRPC3 looks first for an AC-support $\left(x_{j}, v_{j}\right)$ for $\left(x_{i}, v_{i}\right)$ on $c_{i j}$, then it checks if $\left(v_{i}, v_{j}\right)$ is PC. In this last step, changes were necessary to obtain $p$-maxRPC3 (lines 9-12). We check if $\left(v_{i}, v_{j}\right)$ is PC (line 13) only if $\Delta\left(x_{j}, v_{j}\right)$ is smaller than the parameter $p$ (line 9 ).

The propagation phase of maxRPC3 consists in propagating the effect of deletions. While $Q$ is non empty, maxRPC3 extracts a variable $x_{i}$ from $Q$ and checks for each value $\left(x_{j}, v_{j}\right)$ of each neighboring variable $x_{j} \in \Gamma\left(x_{i}\right)$ if it is not maxRPC because of deletions of values in $D\left(x_{i}\right)$. A value $\left(x_{j}, v_{j}\right)$ becomes maxRPC inconsistent in two cases: if its unique PC-support $\left(x_{i}, v_{i}\right)$ on $c_{i j}$ has been deleted, or if we deleted the unique witness $\left(x_{i}, v_{i}\right)$ for a pair $\left(v_{j}, v_{k}\right)$ such that $\left(x_{k}, v_{k}\right)$ is the unique PC-support for $\left(x_{j}, v_{j}\right)$ on $c_{j k}$. So, to propagate deletions, maxRPC3 checks if the last maxRPC support (last known support) of $\left(x_{j}, v_{j}\right)$ on $c_{i j}$ still belongs to the domain of $x_{i}$, otherwise it looks for the next support (algorithm 2). If such a support does not exist, it removes the value $v_{j}$ and adds the variable $x_{j}$ to $Q$. Then if $\left(x_{j}, v_{j}\right)$ has not been removed in the previous step, maxRPC3 checks (algorithm 3) whether there is still a witness for each pair $\left(v_{j}, v_{k}\right)$ such that $\left(x_{k}, v_{k}\right)$ is the $\mathrm{PC}$ support for $\left(x_{j}, v_{j}\right)$ on $c_{j k}$. If not, it looks for the next maxRPC support for $\left(x_{j}, v_{j}\right)$ on $c_{j k}$. If such a support does not exist, it removes $v_{j}$ from $D\left(x_{j}\right)$ and adds the variable $x_{j}$ to $Q$.

In the propagation phase also, we modified maxRPC3 to check if the values are still $p$-maxRPC instead of checking if they are maxRPC. In $p$-maxRPC3, the last $p$-maxRPC support for $\left(x_{j}, v_{j}\right)$ on $c_{i j}$ is the last $\mathrm{AC}$ support if $\left(x_{j}, v_{j}\right)$ is $p$-stable for $\mathrm{AC}$ on $c_{i j}$. If not, it is the last PC support. Thus, $p$-maxRPC3 checks if the last $p$-maxRPC support (last known support) of $\left(x_{j}, v_{j}\right)$ on $c_{i j}$ still belongs to the domain of $x_{i}$. If not, it looks (algorithm 2) for the next AC support $\left(x_{i}, v_{i}\right)$ on $c_{i j}$, and checks if $\left(v_{i}, v_{j}\right)$ is PC (line 9) only when $\Delta\left(x_{i}, v_{i}\right)<p$ (line 8). If no $p$-maxRPC support exists, $p$-maxRPC3 removes the value and adds the variable $x_{j}$ to $Q$. If the value $\left(x_{j}, v_{j}\right)$ has not been removed in the previous phase, $p$-maxRPC3 checks (algorithm 3) whether there is still a witness for each pair $\left(v_{j}, v_{k}\right)$ such that $\left(x_{k}, v_{k}\right)$ is the $p$-maxRPC support for $v_{j}$ on $c_{j k}$ and $\Delta\left(x_{k}, v_{k}\right)<p$. If not, it looks for the next $p$-maxRPC support for $v_{j}$ on $c_{j k}$. If such a support does not exist, it removes $v_{j}$ from $D\left(x_{j}\right)$ and adds the variable $x_{j}$ to $Q$.

\section{Experimental validation of $p$-maxRPC}

To validate the approach of parameterized local consistency, we made a first basic experiment. The purpose of this experiment is to see if there exist problems on which a given level of $p$-maxRPC, with a $p$ uniform on all the constraint network and static during the whole search is more efficient than $\mathrm{AC}$ or maxRPC, or both. 

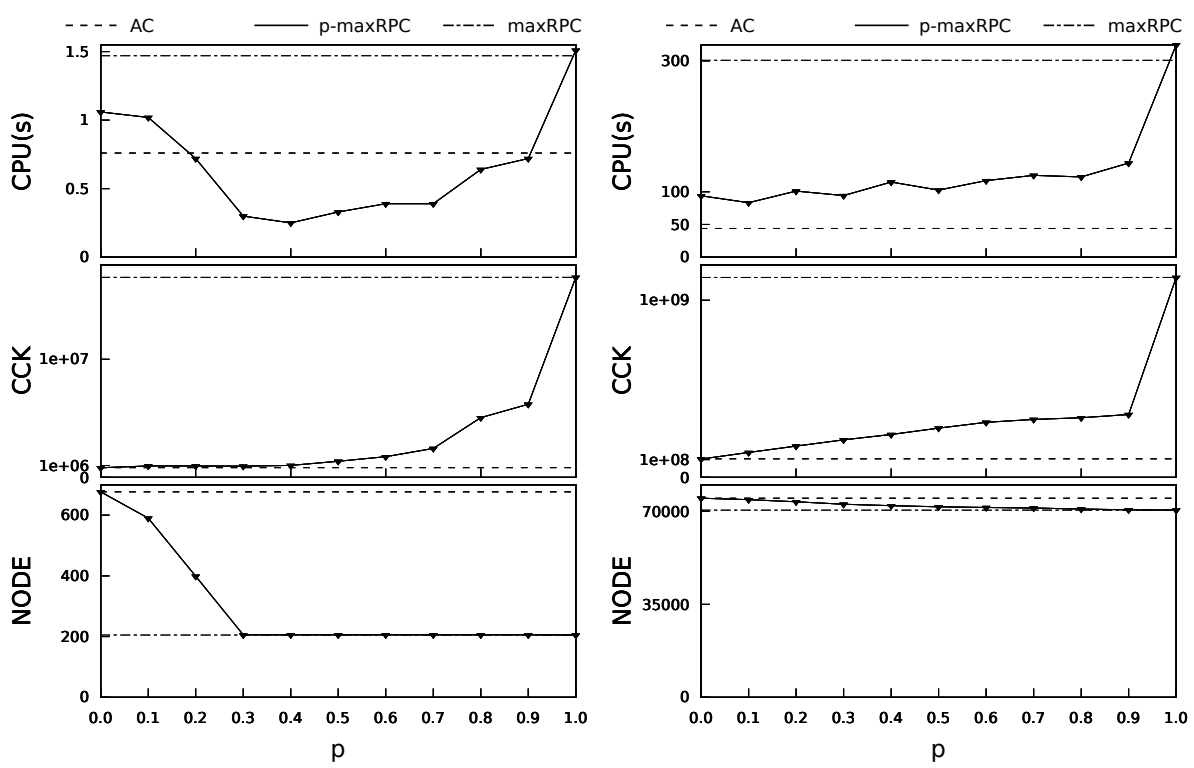

Fig. 2. Instance where $p$-maxRPC outper- Fig. 3. Instance where AC outperforms $p$ forms both $\mathrm{AC}$ and maxRPC. maxRPC.

We have implemented the algorithms that achieve $p$-maxRPC as described in the previous section in our own binary constraint solver, in addition to maxRPC (maxRPC3 version [1]) and AC (AC2001 version [3]). All these algorithms are maintained during search. We tested these algorithms on several classes of problems of the International Constraint Solver Competition $09^{1}$. We have only selected problems involving binary constraints. To isolate the effect of propagation, we used the lexicographic ordering for variables and values. We set the CPU timeout to one hour. Our experiments were conducted on a 12-core Genuine Intel machine with $16 \mathrm{~Gb}$ of RAM running at $2.92 \mathrm{GHz}$.

On each instance of our experiment, we ran $\mathrm{AC}$, max-RPC, and $p$-maxRPC for all values of $p$ in $\{0.1,0.2, \ldots, 0.9\}$. Performance has been measured in terms of CPU time in seconds, the number of visited nodes (NODE) and the number of constraint checks (CCK). Results are presented as "CPU time $(p)$ ", where $p$ is the parameter for which $p$-maxRPC gives the best result.

Table 1 reports the performance of $\mathrm{AC}$, maxRPC, and $p$-maxRPC for the value of $p$ producing the best CPU time, on Radio Link Frequency Assignment Problems (RLFAPs), Geom problems, and queens knights problems. The CPU time of the best algorithm is highlighted with bold. On RLFAP and Geom, we observe the existence of a parameter $p$ where $p$-maxRPC is faster than both $\mathrm{AC}$ and maxRPC for most instances of these two classes of problems. On the queensknight problem, however, AC is always the best algorithm. In Figures 2 and 3, we

\footnotetext{
${ }^{1}$ http://cpai.ucc.ie/09/
} 
Table 1. Performance (CPU time, nodes and constraint checks) of AC, $p$-maxRPC, and maxRPC on various instances.

\begin{tabular}{llrrr} 
& & AC & $p$-maxRPC & maxRPC \\
\hline scen1-f8 & CPU(s) & Time-out & $\mathbf{1 . 3 9}(0.2)$ & 6.10 \\
& \#nodes & - & 927 & 917 \\
& \#ccks & - & $1,397,440$ & $26,932,990$ \\
\hline scen2-f24 & CPU(s) & Time-out & $\mathbf{0 . 1 3}(0.3)$ & 0.65 \\
& \#nodes & - & 201 & 201 \\
& \#ccks & - & 296,974 & $3,462,070$ \\
\hline scen3-f10 & CPU(s) & Time-out & $\mathbf{0 . 8 9}(0.5)$ & 2.80 \\
& \#nodes & - & 469 & 408 \\
& \#ccks & - & 874,930 & $13,311,797$ \\
\hline \hline geo50-20-d4-75-26 & CPU(s) & 111.48 & $17.80(1.0)$ & $\mathbf{1 5 . 0 7}$ \\
& \#nodes & 477,696 & 3,768 & 3,768 \\
& \#ccks & $96,192,822$ & $40,784,017$ & $40,784,017$ \\
\hline geo50-20-d4-75-43 & CPU(s) & $1,671.35$ & $\mathbf{1 , 2 6 4 . 3 6}(0.5)$ & $1,530.02$ \\
& \#nodes & $4,118,134$ & 555,259 & 279,130 \\
& \#ccks & $1,160,664,461$ & $1,801,402,535$ & $3,898,964,831$ \\
\hline geo50-20-d4-75-46 & CPU(s) & $1,732.22$ & $\mathbf{3 7 1 . 3 0}(0.6)$ & 517.35 \\
& \#nodes & $3,682,394$ & 125,151 & 64,138 \\
& \#ccks & $1,516,856,615$ & $584,743,023$ & $1,287,674,430$ \\
\hline geo50-20-d4-75-84 & CPU(s) & 404.63 & $\mathbf{0 . 4 4}(0.6)$ & 0.56 \\
& \#nodes & $2,581,794$ & 513 & 333 \\
& \#ccks & $293,092,144$ & 800,657 & $1,606,047$ \\
\hline \hline queensKnights10-5-add CPU(s) & $\mathbf{2 7 . 1 4}$ & $30.79(0.2)$ & 98.44 \\
& \#nodes & 82,208 & 81,033 & 78,498 \\
& \#ccks & $131,098,933$ & $148,919,686$ & $954,982,880$ \\
\hline \#ueensKnights10-5-mul CPU(s) & $\mathbf{4 3 . 8 9}$ & $83.27(0.1)$ & 300.74 \\
& \#nodes & 74,968 & 74,414 & 70,474 \\
& \#ccks & $104,376,698$ & $140,309,576$ & $1,128,564,278$ \\
\hline & & & &
\end{tabular}

try to understand more closely what makes $p$-maxRPC better or worse than AC and maxRPC. Figures 2 and 3 plot the performance (CPU, NODE and CCK) of $p$-maxRPC for all values of $p$ from 0 to 1 by steps of 0.1 against performance of $\mathrm{AC}$ and maxRPC. Figure 2 shows an instance where $p$-maxRPC solves the problem faster than $\mathrm{AC}$ and maxRPC for values of $p$ in the range [0.3..0.8]. We observe that $p$-maxRPC is faster than $\mathrm{AC}$ and maxRPC when it reduces the size of the search space as much as maxRPC (same number of nodes visited) with a number of CCK closer to the number of CCK produced by AC. Figure 3 shows an instance where the CPU time for $p$-maxRPC is never better than both $\mathrm{AC}$ and maxRPC. We see that if the CPU time for $p$-maxRPC is two to three times better than maxRPC, it fails to improve AC because the number of constraint checks performed by $p$-maxRPC is much higher than the number of constraint checks performed by $\mathrm{AC}$, whereas the number of nodes visited by $p$-maxRPC is not significantly reduced compared to the number of nodes visited by AC. From 
these observations, it thus seems that $p$-maxRPC outperforms $\mathrm{AC}$ and maxRPC when it finds a compromise between the number of nodes visited (the power of maxRPC) and the number of CCK needed to maintain (the light cost of AC).

In Figures 2 and 3 we can see that the CPU time for $1-\operatorname{maxRPC}$ (respectively 0 -maxRPC) is greater than the CPU time for maxRPC (respectively AC) although the two consistencies are equivalent. The reason is that $p$-maxRPC performs tests on the distances. For $p=0$, we also explain this difference by the fact that $p$-maxRPC maintains data structures that $\mathrm{AC}$ does not use.

\section{Adaptative Parameterized Consistency: ap-maxRPC}

In the previous section, we have defined $p$-maxRPC, a version of parameterized consistency where the strong local consistency is maxRPC. We have performed some initial experiments where $p$ has the same value during the whole search and everywhere in the constraint network. However, the algorithm we proposed to enforce $p$-maxRPC does not specify how $p$ is chosen. In this section, we propose two possible ways to dynamically and locally adapt the parameter $p$ in order to solve the problem faster than both $\mathrm{AC}$ and maxRPC. Instead of using a single parameter $p$ during the whole search and for the whole constraint network, we propose to use several local parameters and to adapt the level of local consistency by dynamically adjusting the value of the different local parameters during search. The idea is to concentrate the effort of propagation by increasing the level of consistency in the most difficult parts of the instance. We can determine these difficult parts using heuristics based on conflicts in the same vein as the weight of a constraint or the weighted degree of a variable in [5].

\subsection{Constraint-Based $a p$-maxRPC : $a p c-\operatorname{maxRPC}$}

The first technique we propose, called constraint-based ap-maxRPC, assigns a parameter $p\left(c_{k}\right)$ to each constraint $c_{k}$ in $C$. We define this parameter to be correlated to the weight of the constraint. The idea is to apply a higher level of consistency in parts of the problem where the constraints are the most active.

Definition 6 (The weight of a constraint [5]). The weight $w\left(c_{k}\right)$ of a constraint $c_{k} \in C$ is an integer that is incremented every time a domain wipe out occurs while performing propagation on this constraint.

We define the adaptive parameter $p\left(c_{k}\right)$ local to constraint $c_{k}$ in such a way that it is greater when the weight $w\left(c_{k}\right)$ is higher wrt to other constraints.

$$
\forall c_{k} \in C, p\left(c_{k}\right)=\frac{w\left(c_{k}\right)-\min _{c \in C}(w(c))}{\max _{c \in C}(w(c))-\min _{c \in C}(w(c))}
$$

Equation 1 is normalized so that we are guaranteed that $0 \leq p\left(c_{k}\right) \leq 1$ for all $c_{k} \in C$ and that there exists $c_{k_{1}}$ with $p\left(c_{k_{1}}\right)=0$ (the constraint with lowest weight) and $c_{k_{2}}$ with $p\left(c_{k_{2}}\right)=1$ (the constraint with highest weight).

We are ready to define adaptive parameterized consistency based on constraints. 
Definition 7 (constraint-based ap-maxRPC). A value $v_{i} \in D\left(x_{i}\right)$ is constraint-based ap-maxRPC (or apc-maxRPC) on a constraint $c_{i j}$ if and only if it is constraint-based $p\left(c_{i j}\right)$-maxRPC. A value $v_{i} \in D\left(x_{i}\right)$ is apc-maxRPC iff $\forall c_{i j}, v_{i}$ is apc-maxRPC on $c_{i j}$. A constraint network is apc-maxRPC iff all values in all domains in $D$ are apc-maxRPC.

\subsection{Variable-Based $a p$-maxRPC: $a p x$-maxRPC}

The technique proposed in Section 6.1 can only be used on consistencies where the consistency of a value on a constraint is defined. We give a second technique which can be used on constraint-based or variable-based local consistencies indifferently. We instantiate our definitions to maxRPC but the extension to other consistencies is direct. We call this new technique variable-based ap-maxRPC. We need to define the weighted degree of a variable as the aggregation of the weights of all constraints involving it.

Definition 8 (The weighted degree of a variable [5]). The weighted degree wdeg $\left(x_{i}\right)$ of a variable $x_{i}$ is the sum of the weights of the constraints involving $x_{i}$ and one other uninstantiated variable.

We associate each variable with an adaptive local parameter based on its weighted degree.

$$
\forall x_{i} \in X, p\left(x_{i}\right)=\frac{w \operatorname{deg}\left(x_{i}\right)-\min _{x \in X}(w \operatorname{deg}(x))}{\max _{x \in X}(\operatorname{wdeg}(x))-\min _{x \in X}(\operatorname{wdeg}(x))}
$$

As in Equation 1, we see that the local parameter is normalized so that we are guaranteed that $0 \leq p\left(x_{i}\right) \leq 1$ for all $x_{i} \in X$ and that there exists $x_{k_{1}}$ with $p\left(x_{k_{1}}\right)=0$ (the variable with lowest weighted degree) and $x_{k_{2}}$ with $p\left(x_{k_{2}}\right)=1$ (the variable with highest weighted degree).

Definition 9 (variable-based ap-maxRPC). A value $v_{i} \in D\left(x_{i}\right)$ is variablebased ap-maxRPC (or apx-maxRPC) if and only if it is value-based $p\left(x_{i}\right)$ maxRPC. A constraint network is apx-maxRPC iff all values in all domains in $D$ are apx-maxRPC.

\section{Experimental Evaluation of $a p$-maxRPC}

In Section 5 we have shown that maintaining a static form of $p$-maxRPC during the whole search can lead to a promising trade-off between computational effort and pruning when all algorithms follow the same static variable ordering. In this section, we want to put our contributions in the real context of a solver using the best known variable ordering heuristic, dom/wdeg, though it is known that this heuristic is so good that it reduces a lot the differences in performance that other features of the solver could provide. We have compared the two variants of adaptive parameterized consistency introduced in Section 6 to AC and maxRPC. 
We ran the four algorithms on radio link frequency assignment problems, geom problems, and queens knights problems.

Table 2 reports some representative results. A first observation is that, thanks to the $d o m / w d e g$ heuristic, we were able to solve more instances before the cutoff of one hour, especially the scen11 variants of RLFAP. A second observation is that $a p c-m a x R P C$ and $a p x-m a x R P C$ are both faster than at least one of the two extreme consistencies (AC and maxRPC) on all instances except scen7w1-f4 and geo50-20-d4-75-30. Third, when apx-maxRPC and/or apc-maxRPC are faster than both AC and maxRPC (scen1-f9, scen2-f25, scen11-f9, scen11f10 and scen11-f11), we observe that the gap in performance in terms of nodes and CCKs between AC and maxRPC is significant. Except for scen7-w1-f4, the number of nodes visited by $\mathrm{AC}$ is three to five times greater than the number of nodes visited by maxRPC and the number of constraint checks performed by maxRPC is twelve to sixteen times greater than the number of constraint checks performed by AC. For the Geom instances the CPU time of the ap-maxRPC algorithms is between $\mathrm{AC}$ and maxRPC, and it is never lower than the CPU time of AC. This probably means that when solving these instances with the $\mathrm{dom} / w \mathrm{deg}$ heuristic, there is no need for sophisticated local consistencies. In general we see that the ap-maxRPC algorithms fail to improve both the two extreme consistencies simultaneously for the instances where the performance gap between $\mathrm{AC}$ and maxRPC is low.

If we compare $a p x$-maxRPC to apc-maxRPC, we observe that although apx$\operatorname{maxRPC}$ is coarser in its design than apc-maxRPC, apx-maxRPC is often faster than apc-maxRPC. We can explain this by the fact that the constraints initially all have the same weight equal to 1 . Hence, all local parameters $a p\left(c_{k}\right)$ initially have the same value 0 , so that apc-maxRPC starts resolution by applying AC everywhere. It will start enforcing some amount of maxRPC only after the first wipe-out occurred. On the contrary, in apx-maxRPC, when constraints all have the same weight, the local parameter $p\left(x_{i}\right)$ is correlated to the degree of the variable $x_{i}$. As a result, apx-maxRPC benefits from the filtering power of maxRPC even before the first wipe-out.

In Table 2, we reported only the results on a few representative instances. Table 3 summarizes the whole set of experiments. It shows the average CPU time for each algorithm on all instances of the different classes of problems tested. We considered only the instances solved before the cutoff of one hour by at least one of the four algorithms. To compute the average CPU time of an algorithm on a class of problems, we add the CPU time needed to solve each instance solved before the cutoff of one hour, and for the instances not solved before the cutoff, we add one hour. We observe that the adaptive approach is, on average, faster than the two extreme consistencies AC and maxRPC, except on the Geom class.

In $a p x$-maxRPC and $a p c$-maxRPC, we update the local parameters $p\left(x_{i}\right)$ or $p\left(c_{k}\right)$ at each node in the search tree. We could wonder if such a frequent update does not produce too much overhead. To answer this question we performed a simple experiment in which we update the local parameters every 10 nodes only. We re-ran the whole set of experiments with this new setting. Table 4 reports 
Table 2. Performance (CPU time, nodes and constraint checks) of AC, variablebased ap-maxRPC ( $a p x$-maxRPC), constraint-based $a p$-maxRPC ( $a p c$-maxRPC), and maxRPC on various instances.

\begin{tabular}{|c|c|c|c|c|c|}
\hline & & $\mathrm{AC}$ & $a p x-\max \mathbf{R P C}$ & $p c-\max \mathbf{R P C}$ & $\max R P C$ \\
\hline \multirow[t]{3}{*}{ scen1-f9 } & $\mathrm{CPU}(\mathrm{s})$ & 90.34 & 31.17 & 33.40 & 41.56 \\
\hline & \#nodes & 2,291 & 1,080 & 1,241 & 726 \\
\hline & \#ccks & $3,740,502$ & $3,567,369$ & $2,340,417$ & $50,045,838$ \\
\hline \multirow[t]{3}{*}{ scen2-f25 } & $\mathrm{CPU}(\mathrm{s})$ & 70.57 & 46.40 & 27.22 & 81.40 \\
\hline & \#nodes & 12,591 & 4,688 & 3,928 & 3,002 \\
\hline & $\#$ ccks & $15,116,992$ & $38,239,829$ & $8,796,638$ & $194,909,585$ \\
\hline \multirow[t]{3}{*}{ scen6-w2 } & $\mathrm{CPU}(\mathrm{s})$ & 7.30 & 1.25 & 2.63 & 0.01 \\
\hline & \#nodes & 2,045 & 249 & 610 & 0 \\
\hline & \#ccks & $2,401,057$ & $1,708,812$ & $1,914,113$ & 85,769 \\
\hline \multirow[t]{3}{*}{ scen7-w1-f4 } & $\mathrm{CPU}(\mathrm{s})$ & 0.28 & 0.17 & 0.54 & 0.30 \\
\hline & \#nodes & 567 & 430 & 523 & 424 \\
\hline & \#ccks & 608,040 & 623,258 & 584,308 & $1,345,473$ \\
\hline \multirow[t]{3}{*}{ scen11-f9 } & $\mathrm{CPU}(\mathrm{s})$ & $2,718.65$ & $1,110.80$ & $1,552.20$ & $2,005.61$ \\
\hline & \#ccks & 103,506 & 40,413 & 61,292 & 32,882 \\
\hline & \#nodes & $227,751,301$ & $399,396,873$ & $123,984,9683$ & $3,637,652,122$ \\
\hline \multirow[t]{3}{*}{ scen11-f10 } & $\mathrm{CPU}(\mathrm{s})$ & 225.29 & 83.89 & 134.46 & 112.18 \\
\hline & \#ccks & 9,511 & 3,510 & 4,642 & 2,298 \\
\hline & \#nodes & $12,972,427$ & $17,778,458$ & $6,717,485$ & $156,005,235$ \\
\hline \multirow[t]{3}{*}{ scen11-f11 } & $\mathrm{CPU}(\mathrm{s})$ & 156.76 & 39.39 & 93.69 & 76.95 \\
\hline & \#ccks & 7,050 & 2,154 & 3,431 & 1,337 \\
\hline & \#nodes & $7,840,552$ & $10,006,821$ & $5,143,592$ & $91,518,348$ \\
\hline \multirow[t]{3}{*}{ scen11-f12 } & $\mathrm{CPU}(\mathrm{s})$ & 139.91 & 69.50 & 88.76 & 61.92 \\
\hline & \#ccks & 7,050 & 2,597 & 3,424 & 1,337 \\
\hline & \#nodes & $7,827,974$ & $11,327,536$ & $5,144,835$ & $91,288,023$ \\
\hline \multirow[t]{3}{*}{ geo50-20d4-75-19 } & $\mathrm{CPU}(\mathrm{s})$ & 242.13 & 553.53 & 657.72 & 982.34 \\
\hline & \#nodes & 195,058 & 114,065 & 160,826 & 71,896 \\
\hline & \#ccks & $224,671,319$ & $594,514,132$ & $507,131,3222$ & $2,669,750,690$ \\
\hline \multirow[t]{3}{*}{ geo50-20d4-75-30 } & $\mathrm{CPU}(\mathrm{s})$ & 0.84 & 1.01 & 1.07 & 1.02 \\
\hline & \#nodes & 359 & 115 & 278 & 98 \\
\hline & $\#$ ccks & 261,029 & 432,705 & 313,168 & $1,880,927$ \\
\hline \multirow[t]{3}{*}{ geo50-20d4-75-84 } & $\mathrm{CPU}(\mathrm{s})$ & 0.02 & 0.09 & 0.05 & 0.29 \\
\hline & \#nodes & 59 & 54 & 59 & 52 \\
\hline & \#ccks & 33,876 & 80,626 & 32,878 & 697,706 \\
\hline \multirow[t]{3}{*}{ queensK20-5-mul } & $\mathrm{CPU}(\mathrm{s})$ & 787.35 & $2,345.43$ & 709.45 & $\overline{\text { Time-out }}$ \\
\hline & \#codes & 55,596 & 40,606 & 41,743 & - \\
\hline & \#ccks & $347,596,389$ & $6,875,941,876$ & $379,826,516$ & - \\
\hline \multirow[t]{3}{*}{ queensK15-5-add } & $\mathrm{CPU}(\mathrm{s})$ & 24.69 & 17.01 & 14.98 & 35.05 \\
\hline & \#codes & 24,639 & 12,905 & 12,677 & 11,595 \\
\hline & $\#$ ccks & $90,439,795$ & $91,562,150$ & $58,225,434$ & $394,073,525$ \\
\hline
\end{tabular}


Table 3. Average CPU time of AC, variable-based ap-maxRPC (apx-maxRPC), constraint-based ap-maxRPC (apc-maxRPC), and maxRPC on all instances of each class of problems tested, when the local parameters are updated at each node

\begin{tabular}{|c|l|r|r|r|r|}
\cline { 3 - 6 } \multicolumn{2}{c|}{} & AC & $a p x$-maxRPC & apc-maxRPC & maxRPC \\
\hline \multirow{3}{*}{ Average(CPU) } & geom & $\mathbf{6 9 . 2 8}$ & 180.57 & 191.03 & 279.30 \\
\cline { 2 - 6 } & scen & 18.95 & 9.63 & $\mathbf{8 . 3 0}$ & 13.94 \\
\cline { 2 - 6 } & scen11 & 810.15 & $\mathbf{3 2 5 . 9 0}$ & 467.28 & 564.17 \\
\cline { 2 - 6 } & queensK & 135.95 & 395.41 & $\mathbf{1 2 1 . 7 5}$ & 610.51 \\
\hline
\end{tabular}

Table 4. Average CPU time of AC, variable-based ap-maxRPC (apx-maxRPC), constraint-based ap-maxRPC (apc-maxRPC), and maxRPC on all instances of each class of problems tested, when the local parameters are updated every 10 nodes

\begin{tabular}{|l|l|r|r|r|r|}
\cline { 3 - 6 } \multicolumn{2}{c|}{} & AC & $a p x$-maxRPC & apc-maxRPC & maxRPC \\
\hline \multirow{3}{*}{ Average(CPU) } & geom & $\mathbf{6 9 . 2 8}$ & 147.20 & 189.42 & 279.30 \\
\cline { 2 - 6 } & scen & 18.95 & $\mathbf{7 . 4 0}$ & 8.86 & 13.94 \\
\cline { 2 - 6 } & scen11 & 810.15 & $\mathbf{3 1 1 . 7 4}$ & 417.97 & 564.17 \\
\cline { 2 - 6 } & queensK & 135.95 & 269.51 & $\mathbf{1 1 7 . 1 8}$ & 610.52 \\
\hline
\end{tabular}

the CPU time average results. We observe that when the local parameters are updated every 10 nodes, the gain for the adaptive approach is, on average, greater than when the local parameters are updated at each node. This gives room for improvement, by trying to adapt the frequency of update of these parameters.

\section{Conclusion}

We have introduced the notion of stability of values for arc consistency, a notion based on the depth of their supports in their domain. We have used this notion to propose parameterized consistency, a technique that allows to define levels of local consistency of increasing strength between arc consistency and a given strong local consistency. We have instantiated the generic parameterized consistency approach to max restricted path consistency. We have experimentally shown that the concept of parameterized consistency is viable. Then we have introduced two techniques which allow us to make the parameter adaptable dynamically and locally during search. We have evaluated these two techniques experimentally and we have observed that adapting the level of local consistency during search using the parameterized consistency concept is a promising approach that can outperform both MAC and a strong local consistency on many problems. 


\section{References}

1. Balafoutis, T., Paparrizou, A., Stergiou, K., Walsh, T.: New algorithms for max restricted path consistency. Constraints 16(4), 372-406 (2011)

2. Bessiere, C.: Constraint propagation. In: Rossi, F., van Beek, P., Walsh, T. (eds.) Handbook of Constraint Programming, chap. 3. Elsevier (2006)

3. Bessiere, C., Régin, J.C., Yap, R.H.C., Zhang, Y.: An optimal coarse-grained arc consistency algorithm. Artif. Intell. 165(2), 165-185 (2005)

4. Bessiere, C., Stergiou, K., Walsh, T.: Domain filtering consistencies for non-binary constraints. Artif. Intell. 172(6-7), 800-822 (2008)

5. Boussemart, F., Hemery, F., Lecoutre, C., Sais, L.: Boosting systematic search by weighting constraints. In: ECAI. pp. 146-150 (2004)

6. Debruyne, R., Bessiere, C.: Domain filtering consistencies. Journal of Artificial Intelligence Research 14, 205-230 (2001)

7. Debruyne, R., Bessière, C.: Some practicable filtering techniques for the constraint satisfaction problem. In: IJCAI (1). pp. 412-417 (1997)

8. Katriel, I., Van Hentenryck, P.: Randomized filtering algorithms. Technical Report CS-06-09, Brown University (June 2006)

9. Sellmann, M.: Approximated consistency for knapsack constraints. In: CP. pp. 679-693 (2003)

10. Stergiou, K.: Heuristics for dynamically adapting propagation in constraint satisfaction problems. AI Commun. 22, 125-141 (August 2009) 